\title{
Serial pattern learning by rats: Transfer of a formally defined stimulus relationship and the significance of nonreinforcement
}

\author{
STEWART H. HULSE and NOAH P. DORSKY \\ The Johns Hopkins University, Baltimore, Maryland 21218
}

\begin{abstract}
Two experiments with rats tested independent predictions from cognitive theories of serial pattern learning. The animals learned to anticipate, as measured by running times in a straight alley, different quantities of food pellets organized into formally defined, five-element serial patterns. In Experiment 1, for some animals the patterns were all formally structured according to a monotonic "less than" relationship in which any quantity was always less than its predecessor. For others, no consistent formal rule was applied. Results of a transfer test with a new pattern showed positive transfer if the formal structure of the new pattern was identical to that used initially, but negative transfer if the new pattern was formally different. In Experiment 2, two groups learned the monotonic patterns 18-10-6-3-1 or 18-10-6-3-0 food pellets, while two others learned the nonmonotonic patterns $18-3-6-10-1$ or $10-3-6-10-0$ food pellets. We asked if the difference in value of the terminal element, 1 or 0 food pellets, would affect the facility with which the patterns were learned. The results showed that learning rate and the qualitative response to the elements of the monotonic and nonmonotonic patterns were independent of the value of the terminal element. Both experiments lend additional support to the utility of using cognitive models of human serial-pattern learning for an analysis of the sequential behavior of nonhuman animals.
\end{abstract}

This article results from a line of work testing the feasibility and parsimony of analyzing serial learning in animal behavior with some of the models and techniques developed for serial pattern learning in humans (Hulse, 1978; Hulse \& Dorsky, 1977). In contrast to earlier models of serial learning which stressed chains of stimuli and responses (Hull, 1931; Skinner, 1934), serial pattern learning emphasizes models based on formally expressed relationships among stimuli arranged in sequence.

When people learn serial patterns in the laboratory, they are generally confronted with an organized set of symbols, such as the digit series 123234345 , that they must learn to anticipate, recall, or regenerate item by item. Collections of symbols from which patterns are constructed have been called alphabets (Jones, 1974) or element sets (Hulse, 1978). These may be numbers, as in the foregoing example, lights arranged in linear spatial arrays on a panel (with leftright position coded by number), or letters of the English alphabet. Element sets or alphabets all possess

We thank Carlos Barrerra, Diane Debs, Larry Durban, and David Krunnfusz for their help in data collection. We also thank R. Boakes and N. J. Mackintosh for comments on an earlier version of the manuscript. The research was supported by National Science Foundation Research Grant BMS-73-01400-A02, and by Biomedical Sciences Support Grant 5-S07-RR07041-12 from the Department of Health, Education and Welfare both to The Johns Hopkins University. Reprints may be obtained from Stewart H. Hulse, Department of Psychology, The Johns Hopkins University, Baltimore, Maryland 21218. the common property of an inherent ordered relationship among their several elements, e.g., 2 is greater than 1 , light 4 is to the right of light 3 , the letter $\mathrm{C}$ comes after the letter B, and so on. Furthermore, the ordering is typically well-known by the learner before the experiment begins. Given an alphabet, theories of serial-pattern learning (e.g., Jones, 1974, 1976; Restle, 1970; Simon \& Kotovosky, 1963) then provide a set of primitive combinatorial rules that operate on the elements of the alphabet. When so applied, the rules generate the formally defined structure of a serial pattern. Predictions about the facility with which patterns are learned and remembered then follow from various formal characteristics of one pattern as compared with another. Often the assumption is made that people form an internal representation, perhaps a verbal one, of a pattern's formal structure, and use that representation as a mediator in learning and recalling the pattern. In analogous fashion, rats may form some internal, certainly nonverbal, representation of the stimulus events to which they are exposed. To date, however, we have no idea what that representation, if it exists, might be, and our approach stresses a purely operational analysis of the relation between formally defined serial patterns of stimuli and the behavior they generate.

In our initial work with animals (Hulse, 1978; Hulse \& Dorsky, 1977), we tested the simple hypothesis, directly postulated by any theory of serial 
pattern learning, that formally complex patterns will be more difficult to learn than formally simple patterns. To this end, we constructed serial patterns from an alphabet comprised of a set of food quantities. Food quantity is a stimulus dimension that meets the requirement of an inherent ordering among elements drawn from it. Furthermore, mature rats are familiar with the dimension, and they respond to it differentially in the sense that large quantities of food are chosen over small quantities and generate faster running times (Logan, 1960). The notational system we used for formal characterization of pattern structure represented each element (food quantity) of the pattern as $E(i)$, where the subscript (i) denotes the serial position of element $E$ in the pattern. The formal rules relating successive E(i)s were of three types: "less than," "greater than," and "equals." In line with our hypothesis, we found that it required fewer pattern repetitions for a rat to correctly anticipate pattern elements, as measured by fast and slow running times, the less the formal complexity of the pattern. For example, rats learned a monotonic pattern of 14-7-3-1-0 food pellets formally described by a single "less than" rule, $\mathrm{E}(\mathrm{i}+1)<\mathrm{E}(\mathrm{i})$, faster than a nonmonotonic pattern of 14-1-3-7-0 food pellets formally described by both "greater than" and "less than" rules applied at several points in the pattern. Our results supported the proposition that the rats were somehow differentially sensitive to information in the pattern represented by its formally defined, multielement structure.

The research we report now expands available data relevant to our approach, and provides a further check on the utility and parsimony of our method of analysis. In Experiment 1, we tested hypotheses based on the transfer of a formally defined stimulus relationship from one learning situation to another. In Experiment 2, we tested hypotheses based on the substitution of one pattern element for another in formally defined stimulus structures. The experiments are not closely related in design or conception per se, but both follow simply, directly, and necessarily from any cognitive theory of serial pattern learning.

\section{EXPERIMENT 1}

This experiment was stimulated by the empirical observations that people can generalize formal rules underlying a given serial pattern when they are faced with new or additional sets of elements. Thus, people readily extrapolate a serial pattern of numbers like 1234345 , adding a missing 6 to "fill in the blank" (Simon \& Kotovsky, 1963). Also, Restle and Brown (1970) have shown that people can be biased to apply a particular rule to a pattern that is formally ambiguous by pretraining them with patterns formally structured according to one or another rule. If a pretraining pattern emphasized "trills" of numbers, e.g., 565323787, as compared with "runs" of numbers, e.g., 654765321, then people tended to apply a trill bias in learning a pattern that was ambiguous with respect to trills and runs (e.g., 21223434565). A trill bias led to errors in the fourth position, for example; subjects tended to predict " 1 " instead of " 2 " at that point.

In our experiment, we "biased" rats in the first of two phases by exposing them to appropriate serial patterns of food quantities. The patterns were either decreasing monotonic patterns in which the magnitude of each successive element was described by the "less than" rule, $\mathrm{E}(\mathrm{i}+1)<\mathrm{E}(\mathrm{i})$, or random patterns in which the ordering relation between successive quantities of food was haphazard both within and across patterns. Under each condition, the length of patterns varied haphazardly from pattern to pattern, and so did the absolute value of the food quantities employed. The second, transfer phase of the experiment required the rats in both monotonic and random conditions to learn a new monotonic or nonmonotonic pattern that was fixed in structure and in length.

The effects of pattern structure might appear in one or both of two ways. First, animals trained under conditions described by the consistent monotonic rule might respond to the single consistent feature of the situation and learn to order their running times during initial training, running slower to successive elements regardless of the specific quantities contained in a pattern or the length of a pattern. Animals in the random condition provided a control for any nonassociative effects of multiple runs to various quantities that could result in "patterned" running. Wike and King (1973), for example, reported that during the early part of training, rats ran somewhat slower on successive runs regardless of pattern.

Second, the effects of pattern structure during initial training might reveal themselves through either (or both) positive and negative transfer to the second phase of the experiment. If initial training with monotonic patterns does indeed create a bias to respond to the "less than" relation among food quantities, then animals in this condition should have a relatively easy time mastering a new monotonic pattern in transfer. By the same token, monotonic training might also lead to negative transfer if the new pattern in transfer had a nonmonotonic structure.

\section{Method}

Subjects. The subjects were 24 naive, female albino rats of the Sprague-Dawley strain obtained from ARS/Sprague-Dawley, Madison, Wisconsin, at 80 to 90 days of age. They were maintained in the colony in group cages for $\mathbf{4}$ days after arrival from the supplier and then transferred to ind ividual cages for the duration of the experiment. The rats were randomly assigned to the several experimental conditions.

Apparatus. The apparatus was a straight enclosed runway identical to that used by Hulse and Dorsky (1977). It included a $30-\mathrm{cm}$ 
startbox, a $92-\mathrm{cm}$ alley, and a $30-\mathrm{cm}$ goal area, with the start- and goalboxes separated from the alley by guillotine doors. The goal area contained a $11-\mathrm{cm}$ niche at its far end placed at right angles to the alley. Removable food cups made from bottle caps attached to pieces of wood were located in the niche. Inside width was $10 \mathrm{~cm}$, while inside height was $11 \mathrm{~cm}$ throughout the apparatus. The startand goalboxes were covered with hinged pieces of Plexiglas; the alley proper was covered with hardware cloth. The floor of the apparatus was wood. The start and goal areas were natural wood color, and the alley was painted flat black. A Standard Electric timer started when the startbox door went up and stopped when the rat bridged a $1-\mathrm{cm}$ gap between two metal plates in the goal area. The gap was $20 \mathrm{~cm}$ into the goal area from the guillotine door. Testing was conducted in normal overhead ambient light in a windowless room. Reinforcers consisted of the number of $45-\mathrm{mg}$ Noyes food pellets appropriate for a given run in the apparatus.

Procedure. During initial training, 12 rats were exposed to various monotonically decreasing patterns of reinforcement, while 12 others were exposed to random patterns of reinforcement. Patterns for the monotonic (M) condition were constructed from an element set of $10,5,3,1$, or 0 food pellets. A given pattern could be 4,3 , or 2 elements in length, and the elements of the pattern could be drawn from any in the set, with the restrictions that (a) each element in a pattern be less than its predecessor, and (b) the 1-0 sequence never be used. The latter restriction was applied because given a 1-pellet element in any position in a pattern except the last, 0 pellets had to follow inevitably. One 4-element pattern, three 3element patterns, and three 2-element patterns were used in haphazard order each day, with the order of pattern lengths varying from day to day. All possible patterns were sampled from the complete set shown in Table 1 at least once over the 10 days of initial training. In general, initial training for the $M$ condition was planned such that the only consistent feature from pattern to pattern both within and between days was the consistency of the $E(i+1)<E(i)$ rule for consecutive elements of each sequence.

For the random $(R)$ condition of initial training, pattern lengths, the order in which pattern lengths occurred within a day, and the elements used from pattern to pattern were identical to those used for the $M$ condition, but quantities within patterns sometimes increased, sometimes decreased, and sometimes both increased and decreased. Thus, the only differences between the $M$ and $R$ training conditions were that no consistent ordering relationship was maintained from one element to the next of any given pattern for the $\mathbf{R}$ condition, and the $\mathbf{R}$ condition provided more than one rule governing element-to-element transitions.

The $R$ condition was intended as a control for the effects of practice and exposure to the several elements and pattern lengths used in the initial phase of the experiment. On these grounds it is "neutral" with respect to our hypotheses about transfer in the groups trained initially with the $M$ condition. On other grounds, however, the $\mathrm{R}$ condition may not be neutral, e.g., if one assumes that exposure to a random sequence of events is, in fact, exposure to a "pattern" in which subjects might, for example, learn to ignore food quantity as a dimension. We are unaware of any such theoretical statement directly applicable to our experimental problem, but a number of analogies exist (e.g., Mackintosh, 1975). Because our results speak to it, we return to this problem in due course.

For the second, transfer phase of the experiment, half the rats in the $M$ and $R$ condition of initial training learned a new, fixed mono-

Table 1

Patterns of Food Quantities (Pellets) Used in the Monotonic Conditions of Initial Training

\begin{tabular}{ccccc}
\hline Four-Element & Three-Element & \multicolumn{3}{c}{ Two-Element } \\
\hline $10-5-3-1$ & $10-5-3$ & $10-5$ & $5-3$ & $3-1$ \\
$10-5-3-0$ & $10-5-1$ & $10-3$ & $5-1$ & $3-0$ \\
& $10-3-1$ & $10-1$ & $5-0$ & \\
& $10-3-0$ & $10-0$ & & \\
\hline
\end{tabular}

tonic (M) pattern of 16-9-3-1-0 pellets, while the other half learned a new, fixed nonmonotonic (NM) pattern of 16-1-3-9-0 pellets. The patterns remained fixed in structure from exposure to exposure and from day to day for a total of 25 repetitions, 5 per day. During transfer, then, there were four conditions ( $n=6$ in each): $M-M$, R-M, M-NM, and R-NM, where the first letter designates the treatment applied during initial training, and the letters after the hyphen designate the treatment applied during transfer. Note that both patterns used during transfer differed from any used during initial training, not only in that they were fixed from exposure to exposure, but also in that they were longer by 1 element, and contained a new element, 16 pellets. Thus, while training and transfer patterns shared some features in common, there were some marked differences between them.

Rats were tamed by daily handling and reduced to $85 \%$ of ad-lib body weight over a period of 7 days; they then explored the apparatus for 2 days. On the first day, the animals were brought to the testing room in their home cages and placed in the runway with both guillotine doors open for $10 \mathrm{~min}$. Three food pellets were scattered through the apparatus. On the 2 nd day, each animal received two 5-min exploration periods. Procedures were identical to the first day, except that the food pellets were placed in the food cup in the goalbox.

Following exploration, the rats received 10 days of initial training. They were exposed to 70 patterns (according to the experimental design), 7 patterns per day. Each day, the animals were brought to the testing room in their home cages in groups of three to six. For all runs to each food quantity in a given serial pattern, a rat was placed in the startbox, and the startbox door was raised 3 to $5 \mathrm{sec}$ later. The run ended when the rat stopped the running time clock and finished eating the food pellets (if any). In the case of a nonreinforced run, confinement was for $15 \mathrm{sec}$.

Following a run, the rats were removed to a holding cage to await the start of the next run, which began 10-15 sec later. If a run was the last in a given pattern, the rat was removed to its home cage to await the start of runs to the food quantities in the next pattern, which began 10-20 min later. Water was available in the home cage, but not in the holding cage.

For the first 2 days of initial training, the rats were placed by hand into the goal area if they failed to reach it in $180 \mathrm{sec}$. For the remaining days of the experiment, the animals were allowed $30 \mathrm{sec}$ to reach the goal area on any run. If they failed to meet this criterion, they were removed from the apparatus and placed into the holding cage or home cage to await the start of the next run. The animals were fed a supplementary ration of Purina Laboratory Chow in their home cages in the colony room 15 to $30 \mathrm{~min}$ after testing, sufficient to maintain body weight at $85 \%$ of normal.

The procedures employed during the 5 days of the transfer phase of the experiment, which followed initial training directly, were identical to those used during initial training.

\section{Results and Discussion}

Initial training. There was a tendency for running times to slow as rats ran from one element to the next in the decreasing monotonic patterns used during initial training. Table 2 shows this trend among mean running times at the end of initial training for 2-, 3-, and 4-element patterns. Table 2 also shows, however, that the same tendency holds for the random patterns, a result hardly to be expected if the results in the monotonic condition were due to pattern structure. A similar result was noted by Wike and King (1973). True, the trend is somewhat more pronounced for the monotonic condition, and statistical analysis for the 2-element (but not for the 3or 4-element) patterns shows a reliable interaction be- 
Table 2

Mean Running Times (in Seconds) at End of Initial Training in Two-, Three-, and Four-Element Patterns

\begin{tabular}{ccccc}
\hline \multirow{5}{*}{$\begin{array}{c}\text { Number of } \\
\text { Elements }\end{array}$} & 1st & 2nd & 3rd & 4th \\
\cline { 2 - 4 } & \multicolumn{4}{c}{ Monotonic Condition } \\
2 & 1.1 & 1.3 & 1.9 & \\
3 & 1.0 & 1.1 & 1.4 & 1.3 \\
4 & 1.0 & 1.2 & \\
& \multicolumn{4}{c}{ Random Condition } \\
2 & 1.0 & 1.1 & 1.1 & \\
3 & 1.0 & 1.1 & 1.5 & 1.1 \\
4 & 1.1 & 1.1 & 1.5
\end{tabular}

tween Elements and Groups $[F(1,23)=5.56, p<$ .05]. The Elements by Groups interaction becomes even more reliable if the first and second elements are compared combining the data for different pattern lengths $[F(1,22)=9.82, p<.01]$. However, there is nothing attributable to pattern structure that would predict the appearance of any difference in running times among elements in the random condition, and the differences in running times are, overall, rather unimposing.

Transfer. The results provide good evidence that behavior in transfer was modulated by the "less than" relationship among elements used in initial training. Figure 1 shows the development of responding in the monotonic 16-9-3-1-0 pattern and the nonmonotonic 16-1-3-9-0 pattern to the final 0-pellet element, the element previous work has shown to be most sensitive to the effects of learning different pattern structures (Hulse \& Dorsky, 1977). Mean running times in five-trial blocks are plotted for the M-M, R-M, M-NM, and R-NM conditions. The figure reveals that some, but very little, differentiation of responding occurred for any condition over the first few trials of transfer. However, after Trial 5 , the rats in some conditions began to slow their responding to 0 pellets, initiating a trend that enlarged through the remainder of the experiment. In particular, a comparison of the data in Figure 1 for monotonic as compared with nonmonotonic patterns indicates that the experiment replicated the results of Hulse and Dorsky (1977) in that formally simple monotonic patterns were easier to learn than formally complex nonmonotonic patterns. This was true as measured both by the trials on which slow responding to 0 pellets first began to occur and by the magnitude of differentiation of responding to 0 pellets at the end of the experiment. Comparison of the data from Group M-M with those from Group R-M shows that initial training with monotonic patterns produced faster learning of the transfer pattern. Performance of the M-NM group suggests little, if any, differentiation of running times to 0 pellets, and performance under this condition was poorer than under the R-NM condition. The running times of the R-M and R-NM groups lie between the groups trained initially under the $M$ condition and are consistent with the hypothesis that monotonic patterns lead to faster learning than nonmonotonic patterns.

Figure 2 shows mean running times in the $M-M$ group to each of the five elements of the monotonic pattern used during transfer. The data are plotted for successive five-trial blocks of the transfer phase. Results for the M-M group are displayed because these represent the largest degree of differentiation among the elements of a pattern generated by any condition. Data for the R-M group were quite comparable, except that differentiation of running times to the 1 and 0 pellet elements was not as

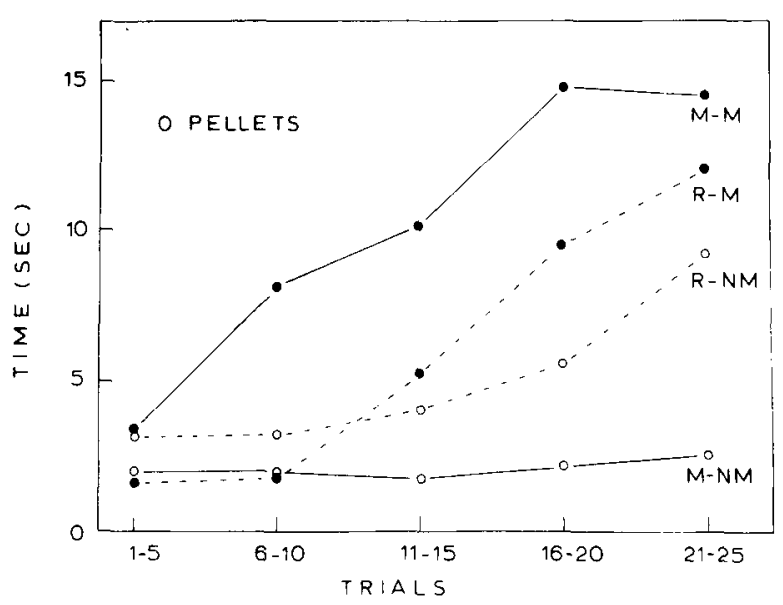

Figure 1. Running times to the 0-pellet element during transfer in Experiment 1. Initial training was with monotonically structured (M) or random (R) patterns. Transfer was to a new monotonic (M) or nonmonotonic (NM) pattern containing 16-9-3-1-0 or 16-1-3-9-0 pellets, respectively.

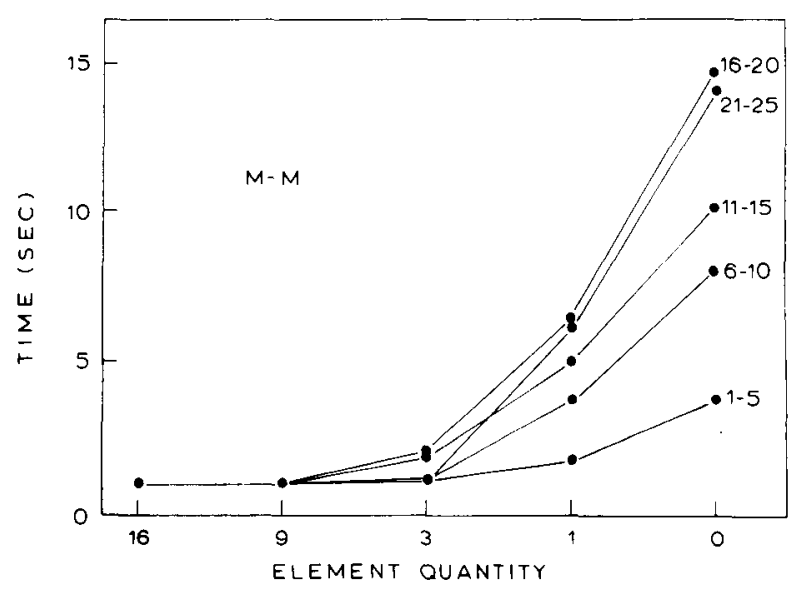

Figure 2. Running times during transfer to the elements of the fixed movotonic 16-9-3-1-0 pattern following initial training with monotonic patteras in Experiment 1. The parameter is blocks of transfer trials. 
large. For the R-NM group, running times to 0 pellets were the only ones to differentiate themselves, while for the M-NM group, there was virtually no suggestion that running times slowed to any elements of the pattern.

The data of Figure 2 indicate that the emergence of slow running times to the progressively smaller quantities of food in the monotonic pattern was a completely orderly affair, with greatest slowing to the 0 -pellet element and progressively less slowing to the 1- and 3-pellet elements. There is little suggestion of differentiation between the 16- and 9-pellet elements, perhaps because the rats were running close to a physiological limit. Differentiation reached an upper asymptote in the M-M group somewhere between Trials 16 and 25 , because the curves for Trials 16 20 and $21-25$ virtually overlap.

An analysis of variance on mean running times supports the conclusions to be drawn from the data of Figures 1 and 2. The analysis showed significant $(p<.001)$ effects for all main effects and all possible interactions, except for the interaction between Days, Groups, and Elements $[\mathrm{F}(48,320)=1.55$, $\mathrm{p}>.05$ ].

Newman-Keuls tests on the means shown in Figure 1 revealed that all groups differed reliably from each other ( $\mathrm{ps}<.05$ or better) in running times to 0 pellets over Trials 16-20 (Day 4). Over Trials 21-25 (Day 5), the same was true except for the differences between the R-M and the M-M groups and the R-M and R-NM groups ( $p s>.05$ ). Over Trials 11-15, all but the R-M and R-NM groups differed reliably.

As a further index of the relative rate of pattern learning in transfer, we assessed when responding to 0 pellets first began to differentiate itself from responding to the other elements in the patterns. To do this, Newman-Keuls tests were run on differences between responding to 0 pellets and responding to 16 pellets. Responding to 16 pellets remained very stable throughout transfer for all groups at $1.0 \pm .2 \mathrm{sec}$. Responding to 0 pellets in the M-M group, but in none of the others, differentiated itself reliably $(p<.01)$ from responding to 16 pellets over Trials 6 10 of transfer. By Trials 11-15, responding to 0 pellets differentiated itself for the R-M group, and by Trials 16-20, the same was true for the R-NM group. The M-NM group never differentiated running times reliably to 0 pellets.

The animals showed more rapid learning of the monotonic pattern-but slower learning of the nonmonotonic patterns-in the transfer phase of the experiment if training in the initial phase was with monotonic as compared with random patterns. The experiment thus provides evidence for positive transfer of the effects of pattern structure when the monotonic pattern in the transfer phase was structurally congruent with the monotonic patterns used initially. Negative transfer appeared, however, if initial and trans- fer pattern structures were not congruent, that is, if experience with monotonic patterns was followed by training with a nonmonotonic pattern.

\section{EXPERIMENT 2}

The generality of the data of Experiment 1 and our earlier experiments on serial pattern learning in animals may be limited by the fact that the research has incorporated a restricted set of food quantities. In particular, most patterns under study have ended with a 0 -pellet quantity. While we have stressed the role of 0 pellets as but one stimulus value along a much broader stimulus dimension of food quantity, 0 pellets provides, of course, the unique condition of nonreinforcement. And nonreinforcement and its attributes play a crucial theoretical and empirical role in many learning phenomena. Given the theoretical and empirical importance of 0 pellets, we have in the past constructed our structurally different patterns such that they all ended with 0 pellets, and we have found it useful to examine the differential responsiveness to 0 pellets as an index of the learning rate associated with patterns of different formal complexity. It seems important, however, to broaden the range of quantities under study and to test the generality of earlier results with patterns which differ in formal complexity but do not contain 0 pellets. This would check on the possibility that earlier results were somehow critically dependent upon the use of 0 pellets per se.

As a second consideration, theories of serial pattern learning hold that patterns of equivalent formal structure are equally easy to learn even though the values of the specific elements comprising them may vary. Thus, people find the number patterns 123234 and 567678 equally easy to memorize because both are built using the same formal next rules, albeit, on different ordered sets of numbers (Simon \& Kotovsky, 1963). In analogous fashion, rats ought to respond similarly to two monotonic patterns which are formally identical in structure but differ in the value of their terminal elements, say 18-106-3-1 and 18-10-6-3-0 food pellets. One might expect that overall quantitative levels of responding might well be faster or slower in patterns which end in 1 as compared with 0 pellets, but relative response times, and thus the qualitative response to successive elements of the patterns which contain the two quantities, ought to be similar. This seems trivially obvious for any number of reasons. However, given the acute salience of nonreinforcement and the fact that element substitution in formally identical patterns is absolutely fundamental to all cognitive theories of serial pattern learning, it seems prudent to make a suitable empirical check.

Finally, an experiment incorporating the monotonic and nonmonotonic patterns noted above should provide a further check on Hulse and Dorsky's 
(1977) contention that while associative explanations may help to account for learning within any given pattern sequence, only structural complexity accurately predicts the relative difficulty of different sequences. In sum, formally simple monotonic patterns should be learned more rapidly than formally complex nonmonotonic patterns, and, moreover, such a result should be independent of the specific values of the elements used.

\section{Method}

Subjects. The subjects were 28 naive, female albino rats, approximately 40-50 days old when obtained from ARS/Sprague-Dawley, Inc., Madison, Wisconsin. They were housed in group cages, 6-8 rats per cage, on ad-lib food and water until they were approximately 90 days of age. At that time they were placed in individual cages and the experiment began. The rats were assigned randomly to the several experimental conditions.

Apparatus. The apparatus was identical to that of Experiment 1.

Procedure. Four groups of seven animals were exposed to repetitions of one of the following 5 element serial patterns of food quantities: 18-10-6-3-1 food pellets (M-1 group), 18-10-6-3-0 food pellets (M-0 group), 18-3-6-10-1 food pellets (NM-1 group), 18-3-6-10-0 food pellets (NM-0 group). The groups thus varied in the complexity of the structure defining the patterns (monotonic as compared with a nonmonotonic ordering) and in the magnitude of the terminal element in the patterns ( 1 as compared with 0 food pellets) in a 2 by 2 factorial design. The values of the elements were chosen such that the total weight of food, and thus hunger reduction, would be virtually identical (within $3 \%$ ) across patterns.

The rats were tamed by daily handling and reduced to $85 \%$ of ad-lib body weight over an initial 7- to 10-day period. Then each explored the apparatus for 3 days, 10 min per day, with food pellets scattered throughout the apparatus.

Ten days of testing began on the day following exploration. On each testing day, the rats were brought in their home cages to the testing room in squads of three to five. Pattern repetitions consisted of one run to each of the five quantities of food in the pattern. Three pattern repetitions were run each day, so over the course of training all animals were exposed to 30 repetitions (150 runs).

A run began when the startbox door went up 1-2 sec following placement of the rat in the startbox. The run ended when the rat finished eating the food in the goalbox. The rats were confined in the empty goalbox for $10 \mathrm{sec}$ when they ran to the 0 -pellet element. If rats on any run failed to reach the goalbox within $60 \mathrm{sec}$, they were removed from the apparatus to await the start of the next run.

The animals were placed in a holding cage for $10 \mathrm{sec}$ between each run of a pattern. During this period, running times were recorded and the apparatus was reset for the next run. The rats were returned to their home cages (which contained water bottles) between repetitions. For any rat, approximately 15-20 min elapsed between the last run of one pattern and the first run of the next.

Supplementary rations of Purina Laboratory Chow were given 20 to $30 \mathrm{~min}$ following daily testing sufficient to maintain the animals at $85 \%$ of ad-lib body weight.

\section{Results and Discussion}

Once behavior stabilized after the 1st day of the experiment, consistent patterns of behavior appeared. The relevant data are plotted in Figures 3 and 4, which show, respectively, mean running times in daily blocks of trials to the initial element and to the terminal elements of the four patterns, and mean running times at asymptote to all 5 elements of the four patterns. The rats slowed their running to the final 1- or 0-pellet element to a much greater extent if that element was part of a monotonic as

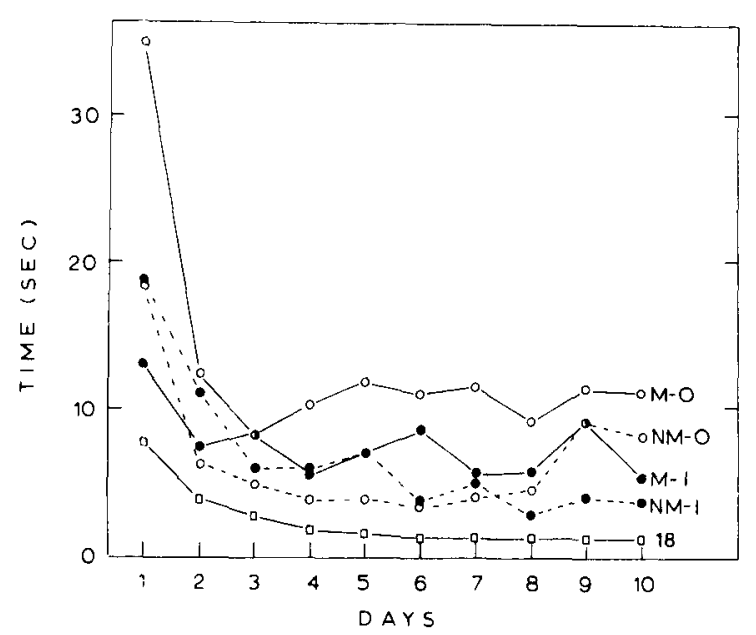

Figure 3. Running times to the initial (18-pellet) and the terminal (1- or 0-peliet) elements of the monotonic (M) or nonmonotonic (NM) patterns. Because they did not differ, the curves for the 18-pellet element have been averaged across conditions.

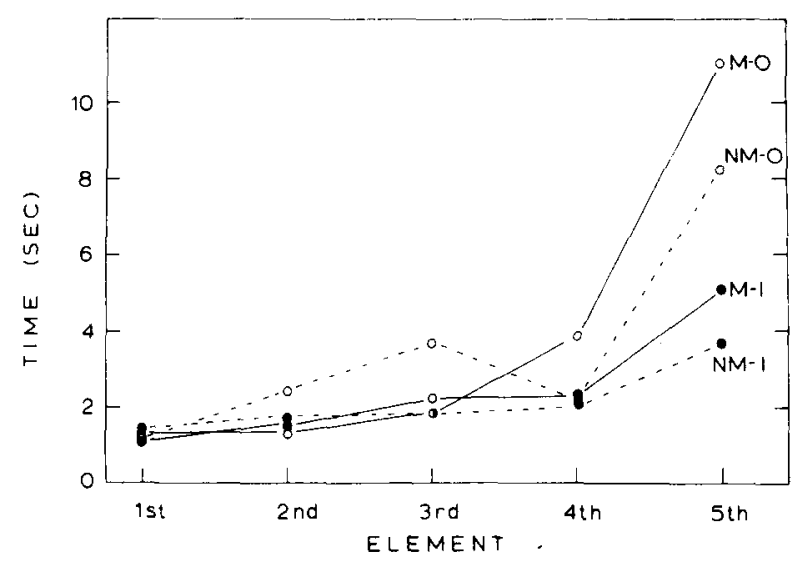

Figure 4. Running times on the last day of the experiment to the 5 elements of the monotonic (M) and nonmonotonic (NM) patterns. All elements within each pattern type were identical except for the last, which was either 0 or 1 pellet.

compared with a nonmonotonic pattern. True, running times were somewhat faster in general to the terminal elements of a pattern if those elements were 1 as compared with 0 pellets, but the qualitative pattern of responding was identical in each case. In particular, patterns containing 1 vs. 0 pellets both led to slow running to the terminal element relative to the earlier elements. Also, the difference in runing times between the terminal elements in monotonic as compared with nonmonotonic patterns was roughly equivalent if the patterns ended with either 1 or 0 pellets.

Statistical analysis supports the reliability of the foregoing results. Analysis of variance on the means for all 10 days of the experiment showed a reliable main effect for quantity alone $[F(4,144)=46.96$, $p<.01$, indicating that larger quantities of food led to faster responding than smaller quantities of food. Collapsing across patterns ending in 1 or 0 pellets, the 
analysis also showed a reliable interaction between pattern structure (monotonic vs. nonmonotonic) and the 5 successive elements in the patterns $[F(4,144)$ $=8.60, \mathrm{p}<.01]$. The Patterns by Elements interaction was also reliable if comparisons were made between just the first 18-pellet quantity (common to all patterns) and the last quantity $[F(1,36)=8.95$, $\mathrm{p}<.01]$. These interactions show that the animals' reaction to the first through the fifth elements was a function of the patterns in which the elements appeared. However, if the value of the terminal element was included as a variable in the analysis, the interactions between pattern structure (monotonic or nonmonotonic) and the value of the 1 - or 0 -pellet terminal element failed to reach significance if all 5 or just the first and last elements were considered $[F(1,36)=3.76$ and 3.87 , respectively, ps $>.05]$. Consequently, there was no reliable evidence that the animals' general reaction to the two pattern structures differed as a function of the value of the terminal element.

Newman-Keuls tests on means for the last day of the experiment associated with the terminal elements in the M-O, NM-O, M-1, and NM-1 conditions showed that all means were reliably different from each other (ps $<.05$ or better) except for the difference between the M-1 and NM-1 patterns. The latter difference was reliable $(p<.05)$ if data were considered for the last 2 days of the experiment combined. Note, however, from Figure 4, that the difference in running times to the last element in the monotonic and nonmonotonic patterns was about the same for both 1- and 0-pellet terminal elementsas the analysis of variance indicated.

Because running times were highly variable on Day 1 of the experiment, the analyses discussed above were run both including and excluding Day 1 data. Identical conclusions were reached in both cases.

Analysis of variance of the means calculated across all three repetitions of Day 1 showed, as Figure 3 indicates, a reliable main effect for terminal element $[F(1,36)=5.32, p<.05]$ and a reliable interaction between pattern structure and terminal element $[F(1,36)$ $=5.72, \mathrm{p}<.05]$. These significant effects were due primarily to the extremely slow responding generated by the 0 -pellet element (especially in the monotonic pattern ending with that quantity), responding that stabilized by the 2 nd day into a patt orn more characteristic of that prevailing for the remainder of the experiment.

Initial differentiation of the terminal element. To determine when responding to the terminal element first began to stably differentiate itself from responding to the initial 18-pellet element, the number of repetitions was counted (excluding those of Day 1) before two out of three successive repetitions occurred in which running times to the terminal quantity were reliably slower $(p<.05)$ than running times to the initial quantity. The appropriate error term from an analysis of variance based on all 30 pattern repetitions of the experiment was used to calculate this critical difference, $3.1 \mathrm{sec}$. Results of the analysis showed that $11.4,17.3,21.0$, and 22.3 repetitions were required to meet the criterion for the $M-0$, M-1, NM-0, and NM-1 groups, respectively. Analysis of variance on these data showed a reliable effect only for monotonic as compared with nonmonotonic patterns $[\mathrm{F}(1,36)=5.67, \mathrm{p}<.05]$. Therefore, although Figure 3 suggests that it took longer for running times to the terminal element in the M-1 and NM-1 conditions as compared with the $\mathrm{M}-0$ and NM-0 conditions to differentiate themselves from running times to 18 pellets, the analysis of pattern repetitions shows, in fact, that this suggestion is misleading.

To summarize, the qualitative response to monotonic and nonmonotonic patterns of food quantities was the same regardless of the value of their terminal elements. Rats responded to successive elements of patterns ending in 1 or 0 pellets with congruent latency changes, and such patterns were learned with equal facility, as measured by the number of pattern repetitions before differential responding to the terminal element began to appear. Thus, there seems nothing to mark a 0-pellet quantity as unique in determining the rat's reaction to pattern structure and, within the limits imposed by the quantities used, the animal's response to pattern structure appeared to depend as much upon the formally defined relations across successive elements as upon any feature associated with the absolute value of the elements employed. The experiment also replicated earlier results of Hulse and Dorsky (1977) by showing that formally simple monotonic patterns were easier to learn than formally complex nonmonotonic patterns.

\section{GENERAL DISCUSSION}

The results of both experiments comply with the general proposition that prompted them-namely, rats are sensitive to formally defined relations among sequences of stimuli in a manner that follows directly from cognitive principles of serial pattern learning. The data of Experiment 1 are especially clear and important in this regard. There the rats behaved in transfer just as one would expect if they were using some internalized analogue of a "less than" rule acquired in initial training for monotonically changing food quantities. The results of Experiment 2 are useful because they show that, given a common formal structure, substitutions can be made for the salient element of nonreinforcement in a pattern without distorting the rat's sensitivity to the pattern's formal properties.

Several aspects of the results warrant elaboration. First, we must entertain, at least, the idea that the random patterns of the initial phase of Experiment 1 were not altogether neutral with respect to pattern structure, and that we should therefore hedge our 
conclusions about transfer because the random patterns were not a suitable control. In the same apparatus under approximately equivalent conditions, Hulse and Dorsky (1977) showed that without initial R training it took no more than four repetitions of a 14-7-31-0 pattern for differentiation of running times to the 0 -pellet quantity to first occur reliably. In the R-M group of the present experiment, it took at least 11 repetitions with the 16-9-3-1-0 pattern in transfer to produce the same result. If this difference is real, it is possible that the relatively rapid learning in the $\mathbf{M}-\mathbf{M}$ as compared with the $\mathrm{R}-\mathrm{M}$ condition may have occurred not because the initial monotonic patterns fostered learning of the new $M$ pattern in transfer, but because the initial random patterns inter iered with learning. This might happen theoretically because the initial random patterns could lead the animals to actively ignore food quantity as a relevant stimulus factor (e.g., Mackintosh, 1975) and they would then have to rediscover its relevance in transfer. However, Hulse and Dorsky also showed that animals required about 40 pattern repetitions to reliably differentiate 0 pellets in their 14-1-3-7-0 pattern, while the present data show that animals in the R-NM condition required 16-20 repetitions to achieve the same thing. At face value, this difference suggests that initial training under the $\mathrm{R}$ condition speeded learning of the NM pattern in transfer, and that is inconsistent with the proposition that the $\mathrm{R}$ patterns led the animals to ignore food quantity as a relevant dimension. Perhaps the differences between earlier results and those we report here are due to differences in the specific food quantities used in the patterns.Also, learning the transfer patterns under all conditions took place after the rats had already had considerable practice in the alley, and they therefore had to learn to produce measurably different running times against a baseline in which they were, in fact, running very rapidly in general. This might have altered the rate at which differential running could appear overtly. Of course, the theoretically "ideal" control would be one in which rats received no training at all during the initial phase and entered transfer completely naive. Practically speaking, even that control is less than ideal, however, because the animals would begin transfer unfamiliar with the apparatus, without practice trials of any kind, and so on. These extraneous factors would certainly modulate overt performance quite apart from factors due to pattern structure per se and so would prevent an unsullied reaction to the new pattern introduced during transfer. Clearly the matter of a suitable control is important, but we find no consistent and compelling evidence which, in the final analysis, suggests that the $R$ condition was an inappropriate baseline for the positive and negative transfer effects due to pattern structure that we obtained.

In contrast to the transfer data, the animals' performance during the initial phase of Experiment 1 provided little evidence that they were responding differentially to pattern structure then. It is not clear why this should be so, but the situation contained (because it was designed to do so) a great deal of uncertainty about when a pattern was to end, what quantities of food given patterns contained, and so on, and the animals may have opted for the not unrealistic performance strategy of running essentially as fast as they could to get all the food with minimal delay. Given that 1 or 0 pellets, which generate especially slow running times, could never be located with accuracy in any pattern, the outcome of the first portion of the experiment is not too surprising. Apparently, the conditions of the transfer phase were required before the bias created by decreasing monotonic patterns could modulate overt performance.

Turning to Experiment 2, there are obvious limits to the conditions under which one could substitute food quantities such that the value of the elements would have little or no effect on the form of the response to formally defined structures of serial patterns. If nothing else, the values of the successive elements must be sufficiently large for the rats to be able to distinguish them, and the limiting case in this regard would also depend upon the relative discriminability of quantities of food pellets at different points along an ordered set of food quantities. While data exist which show that rats can distinguish quantities within the range of those employed here (Hulse, 1978), we know very little about the general metric properties of the dimension of food quantity. We also recognize that our observations about element substitution would acquire more potency if we had a model which made quantitative predictions about running times associated with various aspects of pattern structure. The model remains to be developed. Finally, we note that food (and its absence) for a hungry rat generates a significant emotional state of affairs, and appropriate caution should therefore be employed in generalizing the conclusions we have drawn to other, relatively affect-free dimensions like light, sound, or odor. Whether or not the animal would show similar modes of reaction to serial patterns constructed from such stimuli remains an important empirical question.

\section{Theory}

Two theoretical approaches may be applied to our data. The first, which we outlined in the introduction, is based on principles derived from a cognitive analysis of serial pattern learning. Applying this to Experiment 1, we find that rats can learn and generalize a representation or mediator at least isomorphic to the formal rule, $E(i+1)<E(i)$, we used to construct the monotonic patterns. The representation is independent of the absolute values of the elements used in initial training. As long as elements are discriminable from each other transfer depends only on the rat's having learned some hypothetical 
representation of the formal "less than" relation used to generate the patterns. Given these considerations, the results suggest that the representation for a monotonic decrease in food quantity interacts in additive fashion with the formal complexity of a new pattern to generate the net difficulty encountered in learning the new pattern. Other things being equal, formally simple patterns are easier to learn than formally complex patterns (Hulse \& Dorsky, 1977). The present data show that they are rendered even easier to learn, however, if earlier training biases an animal to apply a "rule" that is congruent with that used to construct the new pattern. By the same token a difficult pattern will be rendered still more difficult if animals come to it with a bias to use a "rule" that is not congruent with the new pattern. In sum, two operational principles predict our results, the concept of formal complexity in pattern structure and the concept of congruency in formal structure between conditions of initial training and those of transfer.

We emphasize again that we use the term "rule" in a strict operational sense to provide a convenient shorthand for the formally defined ordered relationships among entire multielement sets of stimuli in the patterns incorporated in the experiment. We make no specific assumptions at all about the internal representation the rat hypothetically creates for any given operationally defined rule like the monotonic rule we have used. In human serial pattern learning, the assumption is often made, though only rarely explicitly (Estes, 1972), that the representation is verbal and subject to direct rehearsal and recall. As we noted at the outset, we do not believe that rats encode information verbally, but we find it a reasonable idea that other, as yet unexplored, mechanisms may exist that enable the rat to store and utilize information in multielement structures of stimuli. The problem warrants further study, at least, particularly as new evidence accumulates that other nonprimate animals like the pigeon can learn to organize serial patterns of stimuli as multielement sequences. Straub, Seidenberg, Terrace, and Bever (Note 1) found, for example, that pigeons could learn to track arbitrary sequences of key colors in a fashion that argued strongly against any effects due to response feedback from stimulus-response chaining. Instead, the pigeons responded rapidly to the stimulus sequences with a pattern of errors which suggested that they had been encoded as multielement structures.

The second theoretical approach to our data comes from principles of simple association. Looking once again at the results of Experiment 1, there are probably a number of associatively based theories which could be applied. It seems, however, that all would necessarily postulate at least three processes: (1) the direct association of pairs of stimuli (food quantities) in the patterns; (2) stimulus generalization across such stimuli within a pattern; and (3) stimulus gener- alization from the conditions of initial training to those of transfer. Broadly speaking, our results would be predicted by the hypothesis that the animals generalized a series of specific, pairwise, $S_{\text {large }} \rightarrow S_{\text {small }}$ associations among the stimuli of initial training to those of transfer. We can think of other assumptions that might be usefully added, but these seem to be the minimum required.

Given such hypothetical processes, a rat exposed to the monotonic patterns in initial training presumably learned a series of paired relations among neighboring food quantities exhausted by the following set (cf. Table 1): $10 \rightarrow 5,10 \rightarrow 3,10 \rightarrow 1,10 \rightarrow 0$, $5 \rightarrow 3,5 \rightarrow 1,5 \rightarrow 0,3 \rightarrow 1$, and $3 \rightarrow 0$. (Of course remote associations, if any, would extend the list.) By (2) above, many of these associations may also have been modulated by generalization based on stimulus similarities within patterns. Assuming the rat in fact learned all the paired relations in initial training, transfer performance would then be determined, on the basis of (3) above, by two things: direct transfer of stimulus associations any time they were identical throughout the exxperiments, and transfer of generalized stimulus associations any time initial training and transfer stimulus conditions were different-especially those stimulus conditions associated directly with the pattern elements themselves. These propositions adequately predict the form of our results.

We note, however, that learning the new patterns in transfer should have been retarded to the extent that stimulus generalization was a necessary process in the change from initial conditions to those of transfer. Analysis shows that there should be direct transfer any time transfer patterns included $3 \rightarrow 1$ and $3 \rightarrow 0$ pairs, because both of these were also used initially. However, all other pairs, and many other experimental conditions apart from those associated with pattern stimuli, were not identical and should therefore have involved generalization to some greater or lesser extent. First, any associations involving the new elements, 16 and 9 pellets, would involve generalization to the extent that these were discriminably different from initial elements. It is doubtful that 10 and 9 pellets are discriminably different, but Hulse (1978) has shown that 18 pellets are discriminable from 6,1 , or 0 pellets which are, in turn, discriminable from each other. Second, the $1 \rightarrow 0$ pair never appeared initially, yet the elements of this pair generated both the earliest and largest differentiation of running time in transfer. Third, the 5-pellet quantity appeared as the initial member of seven stimulus pairs and the terminal member of five stimulus pairs, i.e., in 12 of the 15 patterns used first. Thus, of 70 initial pattern repetitions, substantially more than half included the 5-pellet stimulus. Yet the 5-pellet quantity never appeared in the patterns of transfer. Finally, the rats were exposed to 1-, 3-, and 4-element patterns initially, but transfer incorporated a new 5- 
element pattern. Taken together, these factors suggest that transfer necessarily involved considerable stimulus generalization-as opposed to direct transfer of specific, already-learned stimulus associations. And that should have worked against the relatively rapid acquisition of the new pattern in the M-M condition that in fact occurred.

Turning to Experiment 2, the results lend themselves at first glance to any theory (e.g., Capaldi, 1967) that stresses the associative conditioning and generalization of sequentially occurring stimulus aftereffects. For example, stimulus generalization or differential development of inhibition and excitation throughout all the elements of patterns, or both, could easily account for the fact that monotonic and nonmonotonic patterns ending in 1 as compared with 0 pellets generated faster overall running times across all pattern elements. To be sure, any such theory would also predict that differentiation of running times to the terminal element should be more laborious in patterns ending in 1 pellet because there should be more generalization (less discrimination) between the terminal element and other pattern elements than in the 0 -pellet case. The data provide no statistically significant evidence for that prediction and argue, in this case, for mediation of the associative process by formally defined structural relations among pattern elements. The strong form of this argument is that structural relations among sequences of stimuli play a prepotent role in the control of sequential behavior. While the data are consistent with the strong form of the argument, they do not force its unconditional acceptance. For example, consider a changed set of stimulus conditions, say a 5-element pattern which is formally monotonic with respect to food quantity but in which the elements are not perceptually different. Clearly, structural relations would not be prepotent in such a situation. The empirical problem then becomes one of determining those conditions under which structural relations do play a role in sequential learning. Experiment 2 demonstrates that the structural effects we have obtained are independent of nonreinforcement. More detailed statements await further analysis and research.

We recognize that our work provides no crucial evidence forcing the adoption of a theory based on serial pattern learning as compared with a theory based on simple associations. Nevertheless, while associative explanations for the results obtained may be adequate, we believe these accounts fail to do proper justice to important facets of the data-as we have argued previously (Hulse \& Dorsky, 1977). For example, conceptualizations based on the formal structure of patterns lead to predictions about the relative ease of pattern learning that are not compelled in any direct fashion by associative theory. We feel that the present data, particularly those of Experiment 1, may raise similar doubts about the potential range of application, if not the accuracy, of associative theory. At the very least, cognitive theories of serial-pattern learning raise issues about pattern learning, the management of sequentially arranged information, and other features of animal intelligence that would not otherwise emerge. Consequently, it seems useful to follow the unique paths for additional investigation that a cognitively oriented approach suggests.

\section{REFERENCE NOTES}

1. Straub, R. O.. Seidenberg, M. S., Terrace. H. S.. \& Bever. T. G. Representation of a sequence by pigeons. Paper read at the meeting of the Psychonomic Society, Washington, D.C.. November 1977.

\section{REFERENCES}

CaPALDI. E. J. A sequential hypothesis of instrumental learning. In K. W. Spence \& J. T. Spence (Eds.), The psychology of learning and motivation (Vol. 1). New York: Academic Press, 1967.

Estes. W. K. An associative basis for coding and organization in memory. In A. W. Melton \& E. Martin (Eds.). Coding processe's in human me'mory. New York: Halsted, 1972.

Husc. C. L. Goal attraction and directing ideas conceived as habit phenomena. Psychological Review, 1931, 38, 487-506.

Hulse, S. H. Cognitive structure and serial pattern learning by rats. In S. H. Hulse, H. Fowler, \& W. K. Honig (Eds.), Cognitive processes in animal behavior. Hillsdale. N.J: Erlbaunı. 1978.

Hulse, S. H., \& DoRSKY, N. P. Structural complexity as a determinant of serial pattern learning. Learning and Motivation, 1977, 8. 488-506.

JoNES. M. R. Cognitive representations of serial patterns. In B. Kantowitz (Ed.). Human information processing: Tutorials in pertormance and cognition. Potomac. Md: Erlbaum, 1974.

Jones. M. R. Time our lost dimension: Toward a new theory of perception, attention and menory. Psychological Review, 1976, 83, 323-345.

Logan, F. A. Incentive. New Haven: Yale University Press, 1960. MACKINTOSH, N. J. A theory of attention: Variations in the associability of stimuli with reinforcement. Psychological Review, 1975, 82. 276.298.

Restle. F. Theory of serial pattern learning: Structural trees. Psychological Review, 1970, 77. 481.495.

Restle. F.. \& Brown, E. R. Serial pattern learning. Journal of Experimental Psychology. 1970, 83, 120-125.

Simon, H. A., \& Kotovsky, K. Human acquisition of concepts for sequential patterns. Psychological Review, 1963. 70. $534-546$

Skinnek. B. F. The extinction of chained reflexes. Proceedings of the National Academy of Sciences, 1934, 20. 234-237.

WIKE, E. L., \& KING, D. D. Sequences of reward magnitude and runway performance. Animal Learning \& Behavior, 1973, I, 175.178 .

(Received for publication June 19, 1978; revision accepted September 18, 1978.) 